\title{
Serum neurotrophin-3 and neurotrophin-4 levels are associated with asthma severity in children
}

\section{To the Editors:}

Asthma is the most common chronic disease in childhood and is characterised by chronic airway inflammation, reversible airflow obstruction and hyperresponsiveness of the airways. Among the substantial pathophysiological aspects of asthma are neuroimmune interaction mechanisms; neurotrophins are mediators of the interactions providing links between immune, structural and neuronal cells.

In recent years, several studies have analysed the effects of neurotrophins on allergic inflammation and airway diseases. Elevated brain-derived neurotrophic factor (BDNF), nerve growth factor (NGF) and neurotrophin-3 (NT-3) levels have been found in bronchoalveolar lavage fluid (BALF) after allergen challenge in allergic asthmatic patients [1, 2]. It has also been shown that asthmatic patients demonstrate elevated levels of neurotrophins (NGF and BDNF) in serum and locally in the airways $[3,4]$, and that allergen provocation significantly increases neurotrophin levels in the airways [2]. Increased neurotrophin (BDNF) concentration also correlated with clinical parameters of allergic airway dysfunction, such as airflow limitation and airway hyperresponsiveness, in asthmatic patients [5], whereas its level is normalised after treatment with inhaled corticosteroids [4]. However, to our knowledge, only one study has investigated neurotrophin (BDNF) levels in a paediatric population of asthmatic patients [6].

We hypothesise that given the relationship between neuronal cells and the immune system in asthma, altered peripheral expression of neurotrophins associated with neuronal dysregulation may affect the course of asthma and disease severity in children.

The present study was performed on a Polish sample of 98 asthmatic patients of Caucasian origin aged from 6 to $18 \mathrm{yrs}$. Patients were recruited from in-patients treated at the Dept of Paediatric Pulmonology, Allergy and Clinical Immunology (Poznan University of Medical Sciences, Poznan, Poland) and from the Outpatient Clinic of Allergology (Provincial Hospital No. 2, Rzeszow, Poland). Asthma diagnoses were made according to the Global Initiative for Asthma (GINA) recommendations, based on clinical asthma symptoms and lung function testing. Spirometry was performed on a LungTest 1000 spirometer (MES, Krakow, Poland) according to European Respiratory Society/American Thoracic Society guidelines [7]. Based on the 2002 GINA guidelines, asthma severity was categorised as mild, moderate or severe $\geqslant 6$ months before inclusion in the study. Atopy was defined according to previously described criteria [8].

The patients were analysed twice: during exacerbation of symptoms and in the asymptomatic period. Exacerbation was defined as the presence of asthma symptoms (daytime symptoms, nocturnal symptoms, limitation of daily activities, the need for reliever treatment and reduced lung function). The asymptomatic period was characterised by good control of asthma symptoms and no acute infections for 2 weeks before blood collection.

All participants and their parents have given written informed consent. The local ethics committee accepted the project.

Concentrations of neurotrophins were measured using the DuoSet $_{\circledR}$ ELISA Development Kit (R\&D Systems, Janki, Poland). For BDNF, serum samples were diluted 1:100 in Reagent Diluent (1\% bovine serum albumin in PBS); for other neurotrophins, undiluted samples were used. The absorbance was read on a plate reader at a wavelength of $450 \mathrm{~nm}$ (Asys UVM 340; Biochrom Ltd, Cambridge, UK). Neurotrophin concentration was quantified against a standard curve calibrated with known amounts of protein. Assays were run in duplicate and the intra-assay variability coefficient was $<5 \%$.

Differences in neurotrophin levels between groups of patients were analysed with the use of nonparametric Kruskal-Wallis tests. Spearman's rank correlation coefficient was used to assess possible relationships between clinical data and neurotrophin concentrations. For comparison of differences in neurotrophin levels between exacerbation and the asymptomatic period, we performed nonparametric Friedman's ANOVA with Kendall's concordance coefficient. A p-value $<0.05$ was considered significant. Calculations were performed using Statistica version 9.0 (StatSoft ${ }_{\circledast}$ Ltd, Milton Keynes, UK).

98 asthmatic children were included in the study. Investigated parameters of lung function testing confirmed significantly lower values for patients analysed during exacerbation compared with the asymptomatic period. The clinical characteristics are shown in table 1.

We did not find significant differences in neurotrophin levels between exacerbation and the asymptomatic period. Serum levels of the analysed neurotrophins correlated with symptoms activity, being higher during exacerbation than in the asymptomatic period of disease, but the difference was not statistically significant.

Analysis of patients according to disease severity revealed that significantly higher levels of NT-3 and NT-4 were observed in patients with moderate and severe asthma compared with mild asthma. BDNF and NGF levels were similar between patients with asthma of different severity, regardless of symptoms activity. The results are shown in table 2.

We also aimed to test whether lung function parameters correlate with neurotrophin levels and found that in the whole group of asthmatic patients, BDNF level was inversely correlated with forced expiratory volume in $1 \mathrm{~s}$. 


\begin{tabular}{|c|c|}
\hline TABLE 1 Character & patients \\
\hline Males & $81(82.6)$ \\
\hline Age yrs & $11.1 \pm 3.8$ \\
\hline \multicolumn{2}{|l|}{ Asthma severity } \\
\hline Mild & $19(19.4)$ \\
\hline Moderate & $55(56.1)$ \\
\hline Severe & $24(24.5)$ \\
\hline Positive SPT & 73.2 \\
\hline Inhaled GC treatment & 97.6 \\
\hline LABA treatment & 66.7 \\
\hline Antileukotriene treatment & 45.3 \\
\hline \multicolumn{2}{|l|}{ FEV $1 \%$ pred } \\
\hline Exacerbation & $81.6 \pm 14.44$ \\
\hline Asymptomatic & $103.8 \pm 87.7$ \\
\hline \multicolumn{2}{|l|}{ FEV $_{1} /$ FVC } \\
\hline Exacerbation & $85.8 \pm 14.5$ \\
\hline Asymptomatic & $96.4 \pm 12.5$ \\
\hline FenO ppb & $33 \pm 43.4$ \\
\hline $\operatorname{IgE} \mathrm{IU} \cdot \mathrm{mL}^{-1}$ & $303.1 \pm 411.5$ \\
\hline
\end{tabular}

Data are presented as $n(\%)$, mean \pm SD or \%, unless otherwise stated. SPT: skin-prick test; GC: glucocorticoid; LABA: long-acting $\beta_{2}$-agonist; FEV1: forced expiratory volume in $1 \mathrm{~s}$; \% pred: \% predicted; FVC: forced vital capacity; FeNO: exhaled nitric oxide fraction; Ig: immunoglobulin.

Assuming previous reports that neurotrophin levels are associated with allergic inflammation underlying asthma, we analysed whether the presence of atopy influenced serum levels of studied neurotrophins. We did not observe any significant differences between patients with allergic asthma compared with those without an atopic background, although in general, neurotrophin levels were higher in patients with allergic asthma.

\begin{tabular}{|c|c|c|c|c|}
\hline \multirow[t]{2}{*}{ TABLE 2} & \multicolumn{4}{|c|}{$\begin{array}{l}\text { Serum neurotrophin levels in patients with } \\
\text { different asthma severity }\end{array}$} \\
\hline & Mild asthma & $\begin{array}{l}\text { Moderate } \\
\text { asthma }\end{array}$ & $\begin{array}{l}\text { Severe } \\
\text { asthma }\end{array}$ & p-value \\
\hline \multicolumn{5}{|l|}{$B D N F \mathrm{pg} \cdot \mathrm{mL}^{-1}$} \\
\hline Exacerbation & 35114.9 & 34275.3 & 36186.7 & 0.868 \\
\hline Asymptomatic & 38224.6 & 39254.0 & 33684.2 & 0.741 \\
\hline \multicolumn{5}{|l|}{$\mathrm{NGF} \mathrm{pg} \cdot \mathrm{mL}^{-1}$} \\
\hline Exacerbation & 6.1 & 22.6 & 34.92 & 0.253 \\
\hline Asymptomatic & 4.4 & 26.5 & 14.9 & 0.139 \\
\hline \multicolumn{5}{|l|}{$\mathrm{NT}-3 \mathrm{pg} \cdot \mathrm{mL}^{-1}$} \\
\hline Exacerbation & 59.8 & 184.1 & 310.4 & 0.145 \\
\hline Asymptomatic & 41.8 & 214.0 & 206.1 & $0.006^{*}$ \\
\hline \multicolumn{5}{|l|}{$\mathrm{NT}-4 \mathrm{pg} \cdot \mathrm{mL}^{-1}$} \\
\hline Exacerbation & 20.1 & 65.8 & 116.1 & 0.020 * \\
\hline Asymptomatic & 18.4 & 67.5 & 65.8 & $0.029 *$ \\
\hline
\end{tabular}

BDNF: brain-derived neurotrophic factor; NGF: nerve growth factor; NT: neurotrophin. ${ }^{*}: p<0.05$.
The main findings of this study are the significantly higher serum NT-4 and NT-3 levels in patients with moderate and severe asthma compared with mild asthmatics, and lack of significant differences in serum neurotrophin levels between exacerbation and the asymptomatic period.

Previous studies have shown that neurotrophins are involved in allergic inflammation in different allergic diseases, including asthma. Local upregulation of neurotrophins was found in BALF from asthmatic patients following allergen exposure [2]. The most extensively studied neurotrophin in regard to allergy and asthma is BDNF. Although it was found that serum BDNF was increased in patients with asthma [4], another study did not confirm that difference [9].

Recently, the first study in a paediatric population of asthmatic patients reported that plasma BDNF levels were significantly higher in paediatric patients with moderate and severe asthma in comparison with controls and mild asthmatic patients [6]. In our study, we did not confirm the previous findings for BDNF level; however, we observed the analogous relationship with asthma severity for two other neurotrophins, NT-3 and NT-4, not analysed in the previous study.

To our knowledge, there are only two studies with comprehensive analysis of serum neurotrophin levels in asthma. The first one was performed by NoGA et al. [4], wherein the authors analysed three neurotrophins (BDNF, NGF and NT-3) in adult allergic asthmatic patients treated or untreated with inhaled steroids, and control subjects. They reported that BDNF and NGF levels were significantly higher in untreated patients with allergic asthma compared to healthy controls and asthmatic patients treated with inhaled corticosteroids. For NT-3, they found that the highest level was observed in asthmatic patients treated with inhaled corticosteroids. The more recent study was performed by KOSKELA et al. [10] in relation to chronic cough. It was found that NGF levels in both serum and sputum were significantly increased only in a subgroup of patients with asthma, whereas BDNF showed no difference between the analysed groups. No studies analysing NT-4 level in regard to asthma have been reported to date, so we could not compare our results to previous findings.

In conclusion, our preliminary results indicated that serum NT-3 and NT-4 levels may be used as potential biomarkers of asthma severity in children. However, further studies including healthy children are necessary to confirm our findings.

\section{A. Szczepankiewicz*,\#, M. Rachel ${ }^{\uparrow}$, P. Sobkowiak", Z. Kycler", I. Wojsyk-Banaszak", N. Schöneich", M. Skibinska ${ }^{+}$and A. Bręborowicz ${ }^{\#}$}

*Laboratory of Molecular and Cell Biology, Dept of Paediatric Pulmonology, Allergy and Clinical Immunology, "Dept of Paediatric Pulmonology, Allergy and Clinical Immunology, Third Dept of Paediatrics, ${ }^{+}$Dept of Psychiatric Genetics, Poznan University of Medical Sciences, Poznan, and 'Outpatient Clinic of Allergology, Provincial Hospital No. 2, Rzeszow, Poland.

Correspondence: A. Szczepankiewicz, Laboratory of Molecular and Cell Biology, Dept of Paediatric Pulmonology, Allergy and 
Clinical Immunology, Poznan University of Medical Sciences, 27/33 Szpitalna St., 60-572 Poznan, Poland. E-mail: alszczep @amp.edu.pl

Support Statement: This study was supported by the Ministry of Science and Higher Education (Warsaw, Poland), grant number N402110534.

Statement of Interest: None declared.

\section{REFERENCES}

1 Undem BJ, Hunter DD, Liu M, et al. Allergen-induced sensory neuroplasticity in airways. Int Arch Allergy Immunol 1999; 118 150-153.

2 Virchow JC, Julius P, Lommatzsch $\mathrm{M}$, et al. Neurotrophins are increased in bronchoalveolar lavage fluid after segmental allergen provocation. Am J Respir Crit Care Med 1998; 158: 2002-2005.

3 Olgart Höglund C, Frossard N. Nerve growth factor and asthma. Pulm Pharmacol Ther 2002; 15: 51-60.

4 Noga O, Hanf G, Schaper C, et al. The influence of inhalative corticosteroids on circulating nerve growth factor, brain-derived neurotrophic factor and neurotrophin-3 in allergic asthmatics. Clin Exp Allergy 2001; 31: 1906-1912.

5 Lommatzsch M, Schloetcke K, Klotz J, et al. Brain-derived neurotrophic factor in platelets and airflow limitation in asthma. Am J Respir Crit Care Med 2005; 171: 115-120.

6 Muller GC, Pitrez PM, Teixeira AL, et al. Plasma brain-derived neurotrophic factor levels are associated with clinical severity in school age children with asthma. Clin Exp Allergy 2010; 40: 1755-1759.

7 Standardization of spirometry, 1994 update. American Thoracic Society. Am J Respir Crit Care Med 1995; 152: 1107-1136.

8 Szczepankiewicz A, Breborowicz A, Sobkowiak P, et al. Polymorphisms of two histamine-metabolizing enzymes genes and childhood allergic asthma: a case control study. Clin Mol Allergy 2010; 8: 14.

9 Joachim RA, Noga $\mathrm{O}$, Sagach $\mathrm{V}$, et al. Correlation between immune and neuronal parameters and stress perception in allergic asthmatics. Clin Exp Allergy 2008; 38: 283-290.

10 Koskela HO, Purokivi MK, Romppanen J. Neurotrophins in chronic cough: association with asthma but not with cough severity. Clin Respir J 2010; 4: 45-50.

\section{Value of s-ACE, BAL lymphocytosis, and CD4+/CD8+ and CD103+CD4+/CD4+ T-cell ratios in diagnosis of sarcoidosis}

\section{To the Editors:}

Sarcoidosis is one of the most common causes of idiopathic lung disease. The international standard for diagnosis of sarcoidosis is based on three criteria: 1) a compatible clinical and/or radiological presentation; 2) histological evidence of noncaseating granulomas; and 3) exclusion of other diseases that produce a similar histological or clinical picture [1]. The diagnosis of sarcoidosis is supported by an elevated CD4+/CD8+ T-cell ratio, bronchoalveolar lavage (BAL) lymphocytosis and elevated serum angiotensin-converting enzyme (s-ACE). However, these parameters are variable in sarcoidosis [2-4].

The integrin CD103 is expressed on CD4+ T-cells in BAL. Due to the high influx of circulating T-cells to the granulomata in sarcoidosis, a reduction in the fraction of BAL CD103+CD4+ T-cells has been suggested as a marker of sarcoidosis, in combination with lymphocytosis and CD4+/CD8+ ratio [5]. In selected groups of patients, decreases in the fraction of CD103+CD4+ T-cells are significantly associated with sarcoidosis [6, 7]. The purpose of this study was to evaluate these parameters as diagnostic markers of sarcoidosis in consecutive patients in a tertiary hospital setting.

The study included patients who underwent BAL with subsequent flow cytometric analyses at the Dept of Respiratory Medicine, Aarhus University Hospital (Aarhus, Denmark) from August 2007 until April 2009 ( $n=107)$. The descriptive parameters and paraclinical findings, including sACE $\left(\mathrm{U} \cdot \mathrm{L}^{-1}\right)$, BAL lymphocytosis (determined by differential count), and CD4+/CD8+ and CD103+CD4+/CD4+ T-cell ratios (determined using flow cytometry), were retrieved from case records. The analysis of CD4+/CD8+ ratio was not performed for five controls. The analysis of CD103+CD4+/CD4+ ratio was not performed for six controls. The lymphocyte count was not performed for 10 controls and two cases. The level of s-ACE was not measured for 34 controls. Two patients in the case group were treated with ACE inhibitors, and measurements of their s-ACE levels were not included in the analyses.

All patients who were included in the case group had biopsyconfirmed sarcoidosis with noncaseating granulomas that were found in transbronchial or mediastinal lymph node biopsies. 11 patients had sarcoidosis radiographic stage $0 / \mathrm{I}$ and eight patients had stage II/III. We did not include patients who were diagnosed with sarcoidosis on the basis of clinical features, radiology or BAL findings alone.

Patients in the control group were diagnosed with other pulmonary diseases $(n=88)$ : extrinsic allergic alveolitis $(n=12)$, idiopathic pulmonary fibrosis $(n=10)$, nonspecific interstitial pneumonitis $(n=6)$, desquamative interstitial pneumonitis $(n=2)$, lymphocytic interstitial pneumonitis $(n=1)$, collagen vascular disease with interstitial lung disease $(n=10)$, unclassified interstitial lung disease $(n=26)$, tuberculosis $(n=2)$, aspergillosis $(n=1)$ and other nongranulomatous lung diseases $(n=18)$. 